\title{
An Acronymic Wilderness
}

The world is now flooded with acronyms and none more so than the world of modern medicine. Acronyms are abbreviations formed from the initial letters of a word or phrase and in medicine are mostly used for disease names (MS, Ml, etc.) as well as for drugs (ARVs, CCBs, etc.) and investigations (MRI, LP, etc.).

I now find that on reading a paper in a medical journal, I come across at least two or more acronyms that I am unable to decipher. I then retrace my steps to somewhere at the beginning of the paper where the object of my desire is written in full (for the first and only time) followed by its acronymic letters, written, almost secretively, in brackets after it.

I have recently come across a treasure trove of acronyms in circulars written as guidelines for working in the primary health care clinics. They are part of the Minister of Health's National Health Insurance Initiative (hereinafter referred to as the MOH's NHII). My knowledge of Human Immuno Viral Medicine needed updating, so I was given the South African Antiretroviral Treatment Guidelines for 2013 and I applaud the authors for putting this subject in the most succinct and readable form possible.

The subject lends itself to an acronymic extravaganza. The authors have not explained or defined them because, firstly, they are now mostly common currency and, secondly, I suspect that they were exhausted after the effort of correlating such a complex nomenclature.

On your behalf, I have recorded in my best obsessive and compulsive way the acronyms in this document, which are:

PMTCT, HIV, BANC, SRH, CCMT, TB, OI, ANC, MTCT, IMCI, DNAPCR, EPI, HCT, ARV, ART, LBW, INH, CTX, CD4, PICT, WHO, FDC, TDF, FTC, 3TC, EFV, AZT, Hb, RPR, NVP, LPV, RTV, ABC, D4T, BF,
PCP, NIMART, RTHB, CHW, DOH, HAART, Rh, STI, TST, IRIS, IPT, FeSO FBC, MCV, WCC, EG, BCG, VL, DLY, NGT, ALT, ASAP, IMCI, IUCD, C/S, and PHC.

It is almost a new hieroglyphic language on its own. By the end of the guidelines, I believe I was approaching SSD (Sensory Semantic Dementia) due to PADAB (Progressive Acronym Disambiguation).

Once one has assimilated acronyms into one's subconscious, they make the reading of an article easier and quicker, but new ones take some time to adapt into one's understanding and may interrupt the flow of sentences.

Acronyms also have to be interpreted in the context within which they have been written as LBW can stand for Low Birth Weight or Leg Before Wicket. Even more care must be taken in medical articles as CHD can stand for Chronic Heart Disease, Congenital Heart Disease or Coronary Heart Disease. As you can imagine with drugs and laboratory investigations, the list of medical abbreviations is now enormous and there is now a Dictionary of Medical Acronyms and Abbreviations. I have, from time to time, come across some informal acronyms in the notes that have usually been recorded by frustrated doctors, such as ETKTM for the patient who has had Every Iest Known Io Man. There is also NFP, which stands for Normal For Pietermaritzburg as well as FOS, which stands, of course, for Full Of...Stool.

In a complex informational world acronyms are here to stay, but we need to find some way of controlling them as they are replicating as rapidly as bacteria, and please remember to RSVP to this article and do it ASAP, OK?

Chris Ellis is a GP in $P M B$, in $K Z N, R S A$.

Email: cristobalellis@gmail.com 